\title{
Kinanthropometry Applications of Depth Camera Based 3D Scanning Systems in Cycling: Repeatability and Agreement with Manual Methods
}

\author{
Alice BULLAS*, Simon CHOPPIN, Ben HELLER, Sean CLARKSON, Jon WHEAT \\ Centre for Sports Engineering Research, Sheffield Hallam University, Sheffield, UK
}

http://dx.doi.org/10.15221/14.290

\begin{abstract}
Recent literature suggests that 2D and 3D anthropometric measures are better predictors of sports performance, than traditional 1D measures. The emergence of 3D scanning systems offers a cheap, easy and effective method of estimating these measures. Therefore the aim of this study was to investigate the repeatability of a depth camera based 3D scanning system, and its agreement with manual methods in the extraction of simple thigh measurements. Using 15 healthy, recreationally active male participants, five measurements of the thigh (upper thigh circumference, mid-thigh circumference, knee circumference, knee to mid-thigh length and mid-thigh to upper thigh length) were taken using an anthropometric tape measure and digital callipers, and scanned using a 4camera Kinect based 3D scanning system (using custom analysis software). Agreement and repeatability was subsequently determined. This study demonstrated a low cost Kinect-based 3D scanning system is capable of extracting length and circumference measures within $\sim 2 \%$ and $\sim 3-4 \%$, respectively, with high repeatability, technical error measurements (TEM) of $\sim 1.80 \%$ and $\sim 0.7 \%$ respectively. The 3D scanning system was able to measure the thigh in good agreement with manual measurement methods, with the presence of systematic bias in circumference. Whilst maintaining a very high degree of repeatability, suggesting it is a suitable method to extract simple thigh measurements.
\end{abstract}

Keywords: Anthropometry, Body Measurement, Kinect, ISAK.

\section{Introduction}

\subsection{General}

Anthropometry is the measurement of the human body to determine its average dimensions, and the proportion of its parts, at different ages and in different races or classes [1]. Various studies have extracted anthropometric measures within sport. Although optimal body dimensions are not the only components necessary for an athlete to excel $[2,3,4]$, many believe it to be an important prerequisite $[5,6]$. The discipline investigating the relationship between anthropometry and sporting performance/movement is termed kinanthropometry [7]. Stewart (2010) [8] suggests kinanthropometry to be the scientific discipline, while anthropometry is a toolbox and skill set. Kinanthropometry assessments are used to improve training practices, identify talent and to assist in understanding the relationship between body size and sports performance $[7,8]$.

Kinanthropometry encompasses one-dimensional (1D), two-dimensional (2D) and three-dimensional (3D) anthropometric measures in addition to body segment inertia parameters (BSIP). Recent literature suggests that more complex $2 \mathrm{D}$ and $3 \mathrm{D}$ anthropometric measures might be better predictors of performance [9], capable of identifying changes in body size and shape, that may otherwise be unnoticeable $[10,11]$. However, the vast majority of literature focuses on the extraction of simple anthropometric measures (height, weight and 1D measures such as segment length and segment girths). A lack of awareness of such measures/ research, and difficulties in extracting such measures are the only justifications for their sparse usage [12], as no study has demonstrated complex anthropometric measures to be unrelated to performance.

The emergence of depth camera scanning systems offer a cheaper, easier and more efficient method of estimating 2D and 3D anthropometric measures and BSIPs [13,14,15,16]. A scanning system allows the collection of kinanthropometry data quickly, reducing the interference with training and performance time. Scanning systems also provide the opportunity to conduct large population studies encompassing all sub-disciplines, distances and performance standards (including elite athletes), rarely examined within the literature [2]. Additionally, Mclean and Parker (1989) [17] suggested athlete kinanthropometry profiles adapt in reaction to rule changes and sport structure, a scanning system would allow such changes to be easily monitored. Additionally, these systems might also improve the reliability and accuracy of body segment inertial parameters as fewer assumptions are made than traditional measurement techniques.

*a.bullas@shu.ac.uk, +44 (0)114 225 5867, http://www.shu.ac.uk/research/cser/ 


\subsection{Kinanthropometry \& Cycling}

Cycling is a sport in which performance is influenced by the static and dynamic properties of the legs, which is influenced by muscle volume [18,19], and thereby potentially also influenced by segment size [20]. This suggests that anthropometric measures may be associated with performance. Cycling is a closed sport, in which the contribution of body shape to performance is greater [2] and it is a sport that utilises anthropometric measures are used in monitoring training, performance and talent identification $[2,3,21]$.

Current kinanthropometry and cycling literature fails to present a coherent view, and predominantly uses simple anthropometric measures [22,23,24,25]. For example, Knechtle et al., (2009) [26] demonstrated a moderate association of anthropometry with the performance of ultra endurance road cyclists, but not with training volume. Yet, Knechtle et al., (2011) [27] revealed training volume, not anthropometry, was related to performance of ultra endurance mountain cyclists. It is suggested that $2 \mathrm{D}$ and $3 \mathrm{D}$ measures may be able to detect subtle changes that are not noticeable by simple measures. For example, Rønnestad et al. (2009) [11] demonstrated thigh strength training improved cycling performance, which correlated with increased thigh muscle cross sectional area (CSA). If only 1D measures (such as thigh circumference) had been taken, no changes in anthropometry would have been apparent. Subsequently, this could cause increases in strength to be incorrectly associated with other factors, such as neurological adaptations. The low sensitivity of simple anthropometric measures might be another reason for the contradictory findings in the literature regarding the relationship between anthropometry and cycling performance. Further investigation and extraction of 2D and 3D measures might provide clarity on the kinanthropometry of cycling, as it has done for other sports. For example, Schranz et al., (2012) [9] demonstrated whole body volume and surface area were the most important predictors of rowing ergometer performance; demonstrating greater importance than traditional predictors of rowing performance: mass, leg length and standing height. However, to use a scanning system in cycling applications, the agreement of the system with traditional methods and its repeatability must first be established. The aim of this study was to investigate the repeatability of a depth camera based three-dimensional scanning system and its agreement with manual methods in the extraction of thigh measurements: thereby determining the suitability of the system to extract simple thigh measures.

\section{Methods}

\subsection{Participants}

Through convenience sampling, 15 healthy, recreationally active male volunteers participated in the study (aged $22 \pm 2.5$ years, height $1.81 \pm 0.06 \mathrm{~m}$, weight $77.90 \pm 10.88 \mathrm{~kg}$ ). Participants were required to be male, over the age of 18 years and able to stand unaided - as all measures were conducted standing. All participants were screened to determine their suitability for participation and required to provide written informed consent. They attended one testing session lasting approximately 60 minutes. All procedures were approved by Sheffield Hallam Ethics Committee.

\subsection{Body Measurement}

This study was a within participant cross over design, in which all participants were measured manually and by the scanning system. A total of five anthropometric measurements of the right leg (Table 1) were taken - chosen because they are traditionally used to determine size of the thigh $[9,11,28,29]$. Each measure was taken three times for each measurement method, and the mean recorded. The anthropometric measurements were chosen. Additionally only the right leg was measured to ensure consistency in current manual method protocols [30].

\subsubsection{Manual Method}

Manual measurements were included as they are the most commonly used anthropometry technique. All manual measurements were taken following the fundamental principles of ISAK (International Society for the Advancement of Kinanthropometry), by a level one accredited ISAK examiner. Not all measures extracted were ISAK standard, and those that were fell outside the remit of a level one examiner; however, the experience of the examiner assisted in the general reliability and repeatability of the measures. Upon arrival, the stature and mass of each participant was measured using a Leicester height stadiometer and digital scales. Each participant had a number of anatomical bony locations palpated and marked on the skin using a coloured eye liner pencil, as detailed in Table 1, to be used as reference points during measurement. All circumference and lengths were measured using a basic anthropometric tape measure (Lufkin Executive Thinline 2m, W606PM) and metal digital callipers (Mitutoyo, Japan) respectively. 
Table 1: Measurements, anatomical locations required and the marker point for each measurement.

\begin{tabular}{lll}
\hline $\begin{array}{l}\text { Anthropometrical } \\
\text { Measurement }\end{array}$ & \multicolumn{1}{c}{ Description } & $\begin{array}{c}\text { Anatomical } \\
\text { Locations }\end{array}$ \\
\hline $\begin{array}{l}\text { Upper-thigh } \\
\text { circumference. }\end{array}$ & $\begin{array}{l}\text { Circumference of the Upper-thigh at 1cm distal to the } \\
\text { gluteal fold site - perpendicular to the long axis [30]. }\end{array}$ & Gluteal fold. \\
$\begin{array}{l}\text { Mid-thigh } \\
\text { circumference. }\end{array}$ & $\begin{array}{l}\text { Circumference of the upper-thigh about the point } \\
\text { equidistant from Trochanterion and Tibiale Laterale [30]. }\end{array}$ & $\begin{array}{l}\text { Trochanterion. } \\
\text { Tibiale Laterale. }\end{array}$ \\
$\begin{array}{l}\text { Knee } \\
\text { circumference. }\end{array}$ & $\begin{array}{l}\text { Circumference of the knee at the midpoint of the posterior } \\
\text { superior border of the patella [30]. }\end{array}$ & $\begin{array}{l}\text { Posterior superior } \\
\text { patella border. }\end{array}$ \\
$\begin{array}{l}\text { Upper-thigh to } \\
\text { mid-thigh length. }\end{array}$ & The vertical distance between upper-thigh to mid-thigh. & $\begin{array}{l}\text { Upper-thigh marker. } \\
\text { Mid -thigh marker. }\end{array}$ \\
$\begin{array}{l}\text { Mid-thigh to knee } \\
\text { length. }\end{array}$ & The vertical distance between mid-thigh to knee. & $\begin{array}{l}\text { Mid-thigh marker. } \\
\text { Knee marker. }\end{array}$ \\
\hline
\end{tabular}

\subsubsection{Scanning Method}

The scanning system consisted of four Microsoft Kinect sensors (Microsoft Cooperation, Redmond, USA), vertically mounted on tripods, approximately $1.28 \mathrm{~m}$ above the ground, at each corner of a $1.44 \mathrm{~m} \times 1.44 \mathrm{~m}$ area (Figure $1 \mathrm{a}, 1 \mathrm{~b}$ ). This created a central capture and calibration volume of $0.4 \mathrm{~m} \times$ $0.4 \mathrm{~m} \times 1.1 \mathrm{~m}$. The system set up was based on previous studies, to ensure the optimum compromise between the number of Kinects and the field of view [15]. To avoid distortion of the depth data, each Kinect was individually calibrated before use, using the method demonstrated in Clarkson et al., 2013 [16]. The scanning system was selected because of its ease of use, low price, portability, and use in previous studies $[15,16,31]$.

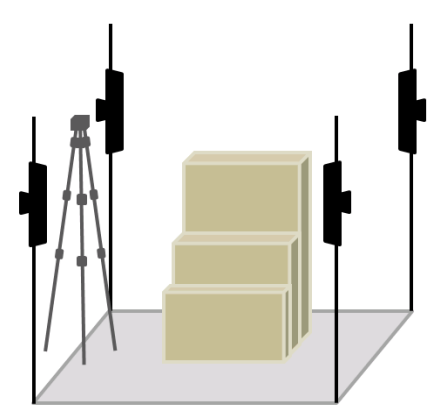

a)

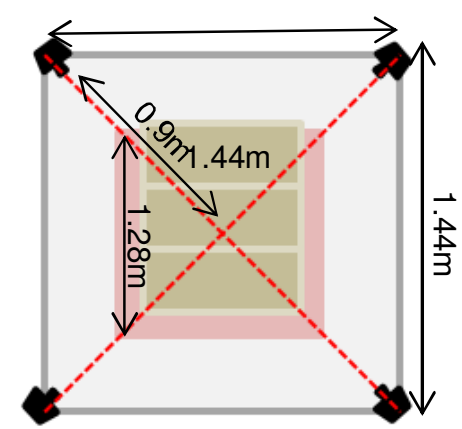

b)

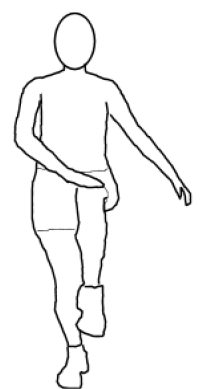

ci)

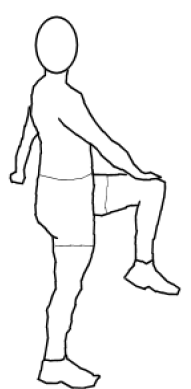

cii)

Figure 1: a) Camera system set-up, b) plan view of system set-up and c) participant scanning position, i) front profile ii) side profile

KinAnthroScan, custom software created in house using Microsoft Kinect software development kit (Microsoft Corporation, Redmond, USA), enabled the calibration of the capture area and the extraction of $3 \mathrm{D}$ and colour data simultaneously, in scans lasting approximately 1.5 seconds. The software calibration procedure followed that detailed in previous studies using this system [15,16,31]: a single scan was taken of the calibration object (4 polystyrene spheres connected by a narrow metal pole) in nine different positions within the calibration volume. The inbuilt calibration procedure identified the sphere centres using Hough transformations and a 3D minimisation technique [15]. These locations were then used to calculate the transformation matrices through rigid body transformations and RANSAC optimisations. Full calibration was conducted at the start of each testing day.

To ensure marker points were visible, coloured self-adhesive circular markers (10mm in diameter) were placed on top of the pencil marker points applied during the manual measurement protocol. To raise the thigh into the calibration volume, avoid occlusion by or measurement of additional limbs, and to ensure analysis of only the relevant points clusters, participants were asked to stand on a raised platform with their left leg lifted and placed on a solid box, placing the knee at $\sim 90^{\circ}$ (Figure 1c), similar to Webster et al., 2013 [34]. The raised platform was necessary to place the thigh in the calibration 
area, as the equipment set-up was fixed, as it was part of a larger data collection study. To assist in the reduction of postural sway, participants were asked to focus on a marker on the wall and provided with a tripod to touch with their left hand $[32,33]$. Participants were reminded to keep the supporting right leg as relaxed as possible to mirror the standing position of manual measures.

A total of four scans were taken of each participant: a practice scan, used to familiarise the participant with the protocol and allow a visual check of the scan position, and three data collection scans, used to extract the five anthropometric measurements. Between scans participants were allowed a small break of $~ 30-60$ seconds, during which participants were required to remain standing and correct their position if necessary. Following the completion of the scans, visual checks were conducted for misaligned data points. Each scan was manually digitised by a single researcher within the KinAnthroScan software and analysis automatically conducted using the same method as previous studies [16]. Assuming the thigh lay perpendicular to the scanning system's global coordinate frame, circumference of the segment was automatically calculated at $2 \mathrm{~mm}$ intervals, producing analysis of thigh circumference over the length of the segment. In addition to producing the length between the digitised points.

\subsection{Analysis}

The calculation of mean differences, absolute and percentage, Interclass Correlation Coefficient (Pearson's Product-Moment Correlation Coefficient) and statistical difference (paired T-test), was used to determine any differences between the two measurement methods. To investigate any differences further, determining the type of error present, Bland-Altman plots were created, alongside the calculation of limits of agreement (LOA) - in addition to Ordinary Least Products Regression (OLP). Technical error of measurement (TEM), absolute and percentage were calculated to determine the repeatability of each measurement method, because of its prevalence when investigating the repeatability manual methods. Microsoft Excel (2010, Microsoft Corporation, USA), MATLAB (version 13.0b, Mathsworks, USA) and SPSS software (version 21.0, IBM, USA) were used to perform this battery of analysis tests.

\section{Results}

Irrespective of the strong positive correlations demonstrated between the two measurement methods, circumference measures exhibited weaker agreement than length measures. All circumference measures demonstrated statistically significant differences between measurement methods $(p<0.05)$, large means differences (4.43 - 2.84\%) and wide limits of agreement (Table 2).

Table 2: The mean absolute $(\mathrm{cm})$ and percentage (\%) difference (mean \pm standard deviation), limits of agreement (LOA), Persons Inter-class Correlation Coefficient (r) and statistical difference (Sig), and 95\% Confidence Interval (Cl).

\begin{tabular}{|c|c|c|c|c|c|c|c|}
\hline \multirow{3}{*}{$\begin{array}{l}\text { Anthropometric } \\
\text { Measure }\end{array}$} & \multirow{2}{*}{\multicolumn{2}{|c|}{$\begin{array}{c}\text { Mean } \\
\text { Difference }\end{array}$}} & \multirow{2}{*}{\multicolumn{2}{|c|}{ LOA $(\mathrm{cm})$}} & \multirow{3}{*}{ r } & \multicolumn{2}{|c|}{ T-Test } \\
\hline & & & & & & \multirow{2}{*}{ Sig. } & \multirow{2}{*}{$\begin{array}{c}95 \% \\
\mathrm{Cl}\end{array}$} \\
\hline & $\mathrm{cm}$ & $\%$ & 1STD & 2STD & & & \\
\hline $\begin{array}{l}\text { Upper-thigh } \\
\text { circumference. }\end{array}$ & $\begin{array}{r}2.74 \\
\pm 1.79\end{array}$ & $\begin{array}{r}4.34 \\
\pm 2.96\end{array}$ & $\begin{array}{r}0.95 \\
-4.52\end{array}$ & $\begin{array}{r}-0.84 \\
-6.31\end{array}$ & 0.92 & 0.00 & $\begin{array}{r}1.86 \\
-3.81\end{array}$ \\
\hline $\begin{array}{l}\text { Mid-thigh } \\
\text { circumference. }\end{array}$ & $\begin{array}{r}1.64 \\
\pm 1.79\end{array}$ & $\begin{array}{r}2.84 \\
\pm 2.23\end{array}$ & $\begin{array}{r}0.40 \\
-2.88\end{array}$ & $\begin{array}{r}-0.83 \\
-4.12\end{array}$ & 0.95 & 0.00 & $\begin{array}{r}1.04 \\
-2.42\end{array}$ \\
\hline $\begin{array}{l}\text { Knee } \\
\text { circumference. }\end{array}$ & $\begin{array}{r}1.61 \\
\pm 0.92\end{array}$ & $\begin{array}{r}3.90 \\
\pm 2.35\end{array}$ & $\begin{array}{r}0.69 \\
-2.53\end{array}$ & $\begin{array}{r}-0.83 \\
-3.44\end{array}$ & 0.95 & 0.00 & $\begin{array}{r}1.15 \\
-2.14\end{array}$ \\
\hline $\begin{array}{l}\text { Mid to upper- } \\
\text { thigh length. }\end{array}$ & $\begin{array}{r}0.18 \\
\pm 0.70\end{array}$ & $\begin{array}{r}2.07 \\
\pm 9.44\end{array}$ & $\begin{array}{l}-0.52 \\
-0.87\end{array}$ & $\begin{array}{r}-1.22 \\
-1.57\end{array}$ & 0.90 & 0.40 & $\begin{array}{r}-0.23 \\
-0.53\end{array}$ \\
\hline $\begin{array}{l}\text { Knee to mid- } \\
\text { thigh length. }\end{array}$ & $\begin{array}{l}-0.17 \\
\pm 1.10\end{array}$ & $\begin{array}{l}-1.56 \\
\pm 7.34\end{array}$ & $\begin{array}{r}-1.27 \\
-0.93\end{array}$ & $\begin{array}{r}-2.37 \\
-2.02\end{array}$ & 0.90 & 0.52 & $\begin{array}{r}-0.76 \\
-0.41\end{array}$ \\
\hline
\end{tabular}

The Bland-Altman plots and the LOA in Figure 2, suggest the type of error demonstrated by the circumference measures to be predominantly systematic, demonstrating good agreement between measurement methods in length measures. 


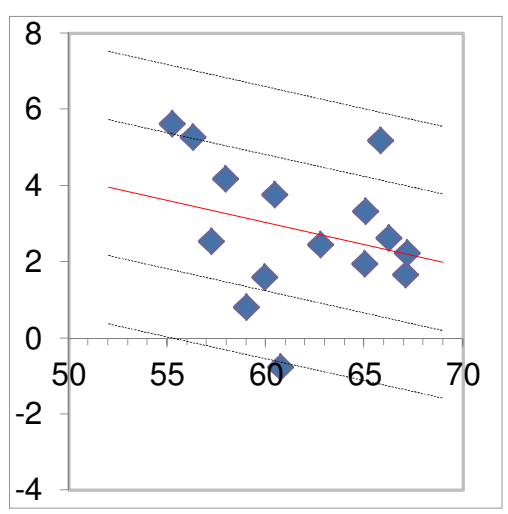

a) Upper-Thigh Circumference

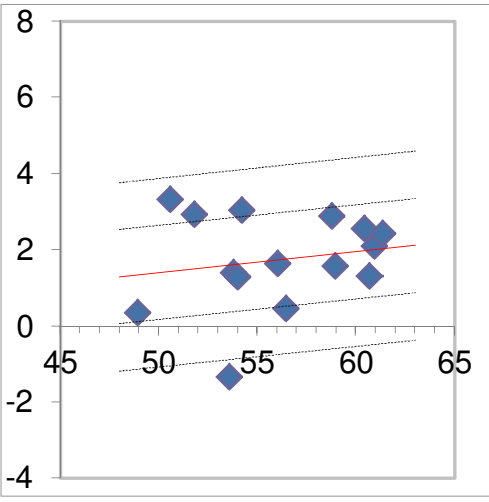

b) Mid-Thigh Circumference

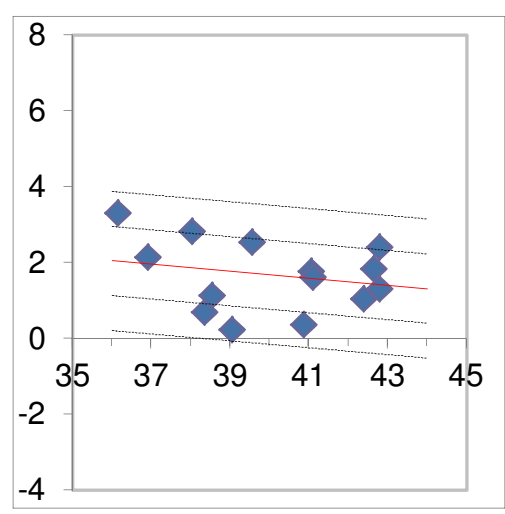

c) Knee Circumference
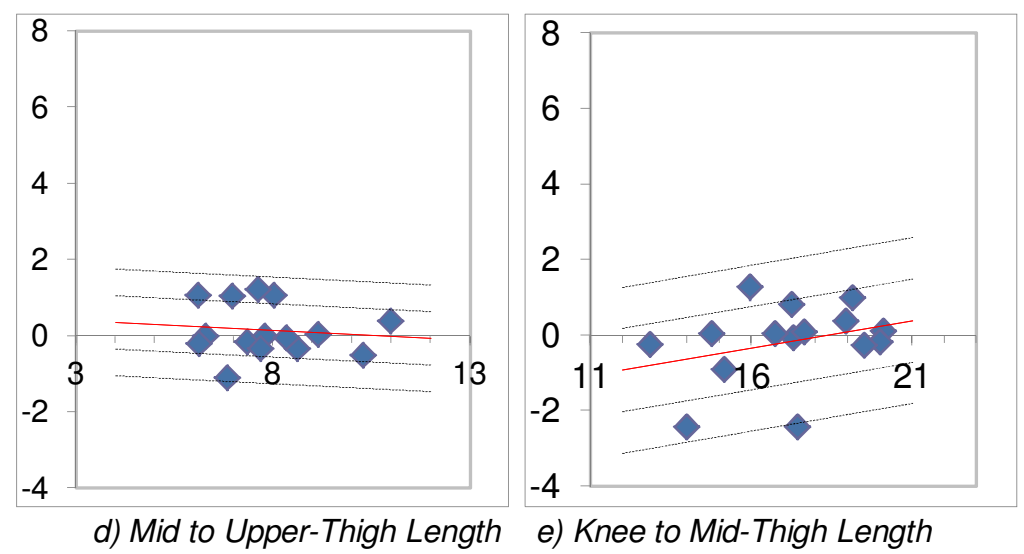

Figure 2: Bland-Altman plots and LOA $( \pm 1 S T D, \pm 2 S T D)$ for each anthropometric measurement between the two methods. $x=$ mean $(\mathrm{cm}), y=$ mean difference $(\mathrm{cm})$.

Although the $95 \% \mathrm{Cl}$ of the OLP analysis lie around 1 and 0, for the intercept (a') and slope (b') $95 \%$ $\mathrm{Cl}$ respectively, the wide $95 \% \mathrm{Cl}$ of intercept (a') (Table 3), suggest the differences demonstrated between measurement methods in circumferences measures is the result of fixed bias. This is reinforced by the OLP plots presented in Figure 3.

Table 3: OLP analysis 95\% Confidence Intervals (95\% Cl) and values between methods.

\begin{tabular}{l|rrr|ccc}
\hline \multicolumn{1}{c|}{ Anthropometric } & \multicolumn{3}{|c|}{ Intercept $\left(\mathrm{a}^{\prime}\right)$} & \multicolumn{3}{c}{ Slope (b') } \\
\multicolumn{1}{c}{ Measure } & Value & \multicolumn{2}{c}{$95 \% \mathrm{Cl}$} & Value & \multicolumn{2}{c}{$95 \% \mathrm{Cl}$} \\
\hline $\begin{array}{l}\text { Upper-thigh } \\
\text { circumference. }\end{array}$ & 9.16 & -3.65 & 21.98 & 0.90 & 0.68 & 1.11 \\
$\begin{array}{l}\text { Mid-thigh } \\
\text { circumference. }\end{array}$ & -1.30 & -11.69 & 9.10 & 1.05 & 0.87 & 1.24 \\
$\begin{array}{l}\text { Knee } \\
\text { circumference. }\end{array}$ & 5.05 & -1.65 & 11.74 & 0.91 & 0.75 & 1.08 \\
$\begin{array}{l}\text { Mid to upper-thigh } \\
\text { length. }\end{array}$ & 0.54 & -1.45 & 2.52 & 0.95 & 0.70 & 1.20 \\
$\begin{array}{l}\text { Knee to mid-thigh } \\
\text { length. }\end{array}$ & -2.73 & -7.84 & 2.39 & 1.15 & 0.85 & 1.45 \\
\hline
\end{tabular}




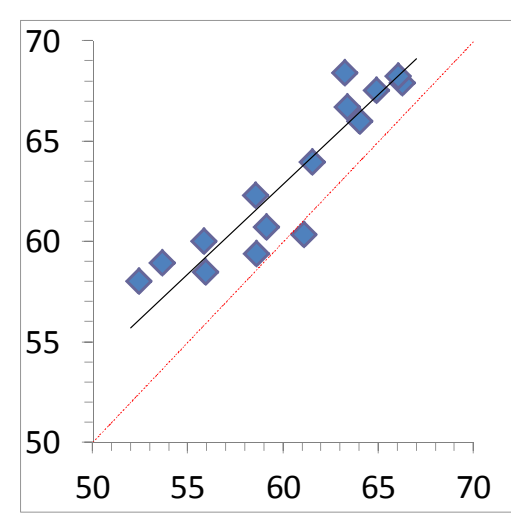

a) Upper-Thigh Circumference

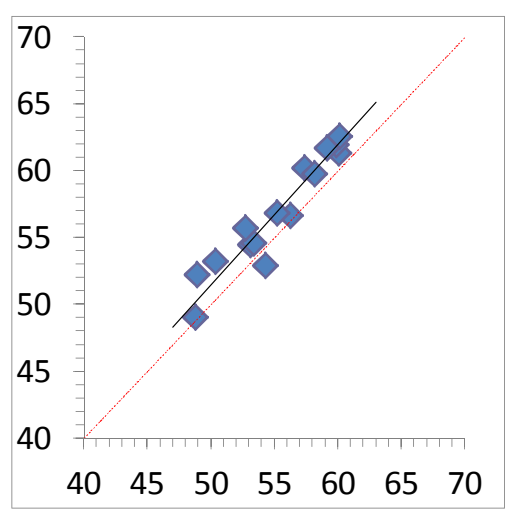

b) Mid-Thigh Circumference

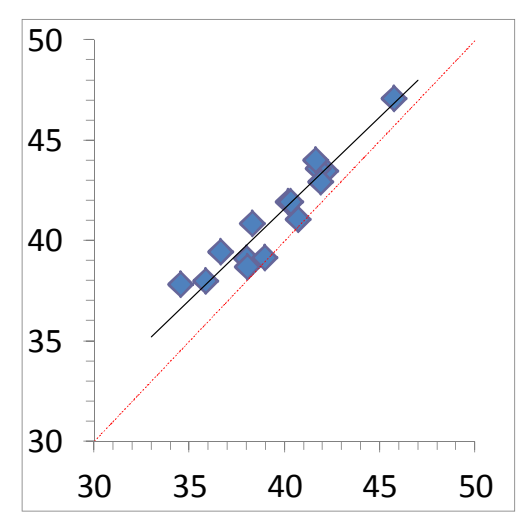

c) Knee Circumference

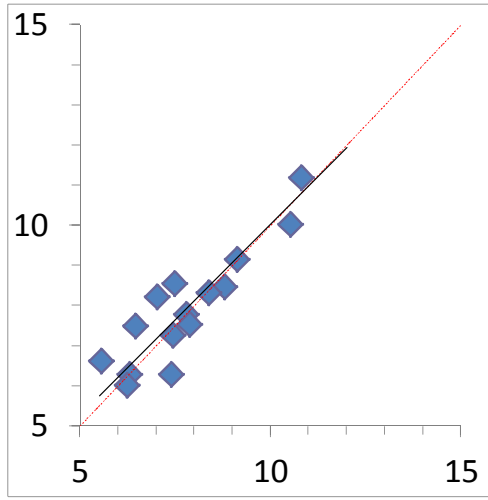

d) Mid to Upper-Thigh Length

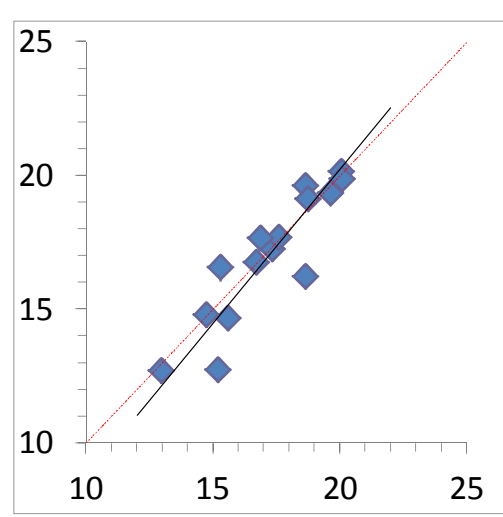

e) Knee to Mid-Thigh Length

Figure 3: OLP plots. $x=$ manual measurement $(\mathrm{cm}), y=$ scan measurement $(\mathrm{cm})$.

The repeatability of both measurement methods appears to be high throughout all anthropometric measurements, demonstrating TEM $<0.7 \%$ and $<1.5 \%$ for circumferences and lengths, respectively (Table 4).

Table 4: Repeatability analysis results for both measurement methods ( $M=$ manual, $S=$ scan): technical error of measurement (TEM); absolute ( $\mathrm{cm}$ ) and relative (\%),

\begin{tabular}{lc|cc}
\hline \multicolumn{1}{c|}{$\begin{array}{c}\text { Anthropometric } \\
\text { Measurement }\end{array}$} & \multicolumn{2}{c}{ TEM } \\
Upper-thigh & $\mathrm{M}$ & 0.35 & 0.57 \\
circumference. & $\mathrm{S}$ & 0.27 & 0.42 \\
Mid-thigh & $\mathrm{M}$ & 0.25 & 0.46 \\
circumference. & $\mathrm{S}$ & 0.19 & 0.33 \\
Knee & $\mathrm{M}$ & 0.22 & 0.55 \\
circumference. & $\mathrm{S}$ & 0.27 & 0.66 \\
Mid to upper-thigh & $\mathrm{M}$ & 0.20 & 1.49 \\
length. & $\mathrm{S}$ & 0.15 & 1.79 \\
Knee to mid-thigh & $\mathrm{M}$ & 0.23 & 0.79 \\
length. & $\mathrm{S}$ & 0.26 & 1.56 \\
\hline
\end{tabular}

\section{Discussion}

Current literature has demonstrated 3D scanning systems are capable of producing repeatable measurements, in close agreement with manual methods $[28,35,36]$. This study has replicated these findings, demonstrating a low cost Kinect-based 3D scanning system is capable of extracting length and circumference measures within $\sim 2 \%$ and $\sim 3-4 \%$ of manual measurements, respectively, with high repeatability, $\sim 1.80 \%$ and $\sim 0.7 \%$ respectively. 
All length measures of the thigh demonstrated non-significant differences between measurement methods. Although this does not automatically imply agreement, good agreement was demonstrated in the Bland-Altman plots, LOA and OLP analysis, in which minimal error is demonstrated.

However, all circumference measures did demonstrate statistically significant differences. Following further investigation, using Bland-Altman plots, the differences appear to be systematic and random due to the wide LOA. However, the systematic and fixed properties of the data appear more confidently demonstrated, OLP analysis that suggests the error to be predominantly fixed and the OLP plots present the majority of data points to lie systematically above the line of identity. Because comparison was only made against manual measurement and not a gold standard it is unclear which method is the cause of the bias. Fixed systematic bias between 3D scanning and manual measurements of circumference is common [16]. Although using a different scanning system, $\mathrm{TC}^{2}$, Zwane et al. (2010) [35] found manual measurements of the body were generally lower than scan measurements, attributing it to the compression of soft tissues during manual measurements. However, literature that focuses on the agreement in length measurements appears to demonstrate greater bias than this study whilst maintaining similar mean differences $[37,38,39]$.

Investigation into the repeatability of the measurement methods demonstrated minor, statistically nonsignificant, differences of $0.7 \%$ and $1.8 \%$, for thigh circumferences and lengths respectively. All circumference measurement taken by the scanning system fall within the ISAK standards, intraexaminer TEM $<1.0 \%$ for all measures taken by level $2-4$ anthropometrists [40], emphasizing the suitability of the scanning method. However, the TEM recorded for length measurements are greater for both manual and 3D scanning measurement methods - the reasons for this are unclear.

Although this study was designed to limit potential sources of error, there are several common methodological limitations: the small sample size, the use of only male participants and the use of the most commonly used method opposed to a gold standard. In addition there are several additional potential sources of error that need to be acknowledged and considered in future investigations. All anthropometric measures were taken standing, with the left leg raised during the scanning protocol to avoid obscuring the right leg. It is possible that the adjustments in position required to maintain balance altered the dimensions of the thigh and the gluteal fold (thereby the upper-thigh anatomical landmark), as well as placing the leg at an angle not perpendicular to the horizontal plane of the global coordinate system, resulting in the extraction of mis-aligned slices and larger circumferences. Although precautions were taken to reduce the postural sway of participants and all scans were visually inspected to check for misalignment, the increased postural sway caused by balancing on one leg may have increased errors in the scan measurement. Additionally, movement of the markers could be a potential source of error. The coloured markers applied for scanning were applied on top of the shorts, and although alignment with the pencil skin marker points was checked, movement could have occurred between scans, resulting in measurement of the incorrect circumference.

Manual measurement using a tape measure is also associated with potential error sources, such as skin compression during measurement [41]. However, a major source of error between the manual measurements and a scanning system is that a tape measure does not directly measure complete skin circumference as the tape does not follow all contours of the segment, skimming over any dimples or indentations [28]. Whereas the scanning, method follows the exact surface distance potentially explaining the overestimation.

\section{Conclusion}

This study demonstrates that a depth camera-based 3D scanning system shows good agreement with manual measurements, accompanied by small systematic error. The 3D scanning system also demonstrated very good repeatability, with TEM less than the ISAK standard of $1.0 \%$.

\section{References}

[1] Oxford English Dictionary (a), anthropometry, [Online]. Available: http://www.oed.com/view/Entry/8444?redirectedFrom=anthropometry\&. [Accessed: June 2014].

[2] E. Wolstencroft, Talent identification and development: an academic review. sportscotland, 2002.

[3] Canadian Cycling Association, Long Term Athlete Development (Volume 1). Canada: Canadian Cycling Association, February 2008. 
[4] L. Brunkhorst and H. Kielstein, "Comparison of anthropometric characteristics in professional triathletes and cyclists." Biology of Sport, vol.30, pp.269-273, 2013.

[5] J. S. Duncan et al. "Pedometer-determined physical activity and body composition in New Zealand children." Medicine and Science in Sports and Exercise, vol.38, pp.1402-1409, 2006.

[6] E. Gualdi-Russo and L. Zaccagni, "Somatotype, role and performance in elite volleyball players." Journal of Sports Medicine and Physical Fitness, vol.41, pp. 256-262, 2001.

[7] A.D. Stewart, "Kinanthropometry - the interdisciplinary discipline." Journal of Sports Sciences, vol.25, no.4, pp.373-373, 2007.

[8] A.D. Stewart, " Kinanthropometry and body composition: A natural home for three-dimensional photonic scanning." Journal of Sports Sciences, vol.28, no.5, pp.455-457, 2010.

[9] N. Schranz et al. "Is three-dimensional anthropometric analysis as good as traditional anthropometric analysis in predicting junior rowing performance?" Journal of Sports Sciences, vol.30, no.12, pp.1241-1248, 2012.

[10] N. Daniell et al." Volumetric Differences in Body Shape Among Adults With Differing Body Mass Index Values: An Analysis Using Three-Dimensional Body Scans." American Journal of Human Biology, vol. 26,no.2, pp.156-163, 2013.

[11] B.R. Rønnestad et al." Effect of heavy strength training on thigh muscle cross-sectional area, performance determinants, and performance in well-trained cyclists." European Journal of applied Physiology, vol.108, pp.965-975, 2010.

[12] M. Sicotte et al. "Reliability of anthropometric measures in a longitudinal cohort of patients initiating ART in West Africa." BMC Medical Research Methodology, vol.10, no.102, 2010.

[13] S. Choppin and J. Wheat, "The potential of the Microsoft Kinect in sports analysis and biomechanics." Sports Technology, vol.6, no.2, pp.78-85, 2013

[14] J. Wheat et al. "Development and assessment of a Microsoft Kinect based system for imaging the breast in three dimensions." Medical Engineering \& Physics, vol.36, no.6, pp.732-738, 2014.

[15] S. Clarkson et al. "Calculating Body Segment Inertia Parameters from a Single Rapid Scan Using the Microsoft Kinect," in $3^{\text {rd }}$ International Conference on 3D Body Scanning Technologies Conference, Lugano, Switzerland, pp.153-163, 2012.

[16] S. Clarkson et al. " Distortion Correction of Depth Data from Consumer Depth Cameras," in $4^{\text {th }}$ International Conference on 3D Body Scanning Technologies Conference, Long Beach, USA, pp. 426-437, 2013.

[17] B.D. McLean and A.W. Parker. "An anthropometric analysis of the elite Australian track cyclist." Journal of Sport Sciences, vol.7, pp. 247-255, 1989.

[18] J. Hopker et al. "Cycling efficiency in trained male and female competitive cyclists." Journal of Sport Science and Medicine, vol.9, pp.332-337, 2010.

[19] L. Dellanini et al. "The Effects of Changing Bone and Muscle Size on Limb Inertial Properties and Limb Dynamics: A Computer Simulation." Computer Methods in Biomechanics and Biomedical Engineering, vol.7, no.3, pp.167-176, 2004.

[20] L. Chen et al. "Thigh muscle volume predicted by anthropometric measurements and correlated with physical function in the older adults." The Journal of Nutrition, Health \& Aging, vol.15, pp. 433-438, 2011.

[21] J.P. Foley et al. "Anthropometric comparison of cyclists from different events." British Journal of sports Medicine, vol.23, pp.30-33, 1989.

[22] D.T.Martin et al. " Physiological characteristics of nationally competitive female road cyclists and demands of competition." Sports Medicine, vol.31, no.7, pp.469-477, 2001.

[23] H. Lee et al. "Physiological characteristics of successful mountain bikers and professional road cyclists" Journal of Sports Sciences, vol.20, pp.1001-1008, 2002.

[24] L. Rossi et al. "Anthropometric and nutritional profile of male mountain bikers". Brazilian Journal of Biomotricity, vol.4, no.3, pp.180-189, 2010. 
[25] C. D. Paton and W. G. Hopkins, "Seasonal changes in power of competitive cyclists: implications for monitoring performance". Journal of Science and Medicine in Sport, vol.8, no.4, pp.375-381, 2005.

[26] B. Knechtle and G. Kohler. "Running performance not anthropometric factors is associated with race success in a Triple Iron Triathlon". British Journal of Sports Medicine, vol.43, pp.437-441, 2009.

[27] B. Knetchlet et al. "Upper body skin-fold thickness is related to race performance in male Ironman triathletes". International Journal of Sports Medicine, vol.32, pp.20-27, 2011.

[28] J. P. Bougard et al. "A comparison of women's sizing by 3D electronic scanning and traditional anthropometry". The Journal of Textile Institute, vol.91, no.2, pp.163-173, 2000.

[29] C. A. Rust et al. "A Comparison of Anthropometric and Training Characteristics among Recreational Male Ironman Triathletes and Ultra-Endurance Cyclists". Chinese Journal of Physiology, vol.55, no.2, pp.114-124, 2012.

[30] International Society For The Advancement Of Kinanthropometry. International Standards for Anthropometric Assessment. The International Society For The Advancement Of Kinanthropometry, Australia, 2011.

[31] S.B. Choppin et al. " Breast Volume Calculation Using a Low-Cost Scanning System,"in 4th International Conference on 3D Body Scanning Technologies, Long Beach CA, USA, pp.19-20, November 2013.

[32] M. Kouzaki and K. Masani,"Reduced postural sway during quiet standing by light touch is due to finger tactile feedback but not mechanical support". Experimental Brain Research, vol. 188, no.1, pp.153-158, 2008.

[33] J. Lackner et al. "Stabilization of posture by precision touch index finger with rigid and flexible ligaments". Experimental Brain Research, vol.139, no.4, pp.454-464, 2001.

[34] J. Webster and J. Cornolo,"Comparison of European and Asian Morphology". International Conference on 3D Body Scanning Technologies Conference, Long Beach, USA, pp.238-242, 2013.

[35] P. E. Zware et al. "A preliminary comparative analysis of 3D body scanner, manually taken girth body measurements and size chart measurements". International Journal of Consumer Studies, vol.34, pp.265-271, 2010.

[36] H. Han et al. "Comparative analysis of 3D body scan measurements and manual measurements of size Korea adult females". International Journal of Industrial Ergonomics, vol.40, pp.530-540, 2010.

[37] T. Catherwood et al. "Validation of a passive stereophotogrammetry system for imaging of the breast: a geometric analysis'. Medical Engineering \& Physics, vol.33, no.8, pp.900-905, 2011.

[38] J. Wheat et al. "Development and assessment of a Microsoft Kinect based system for imaging the breast in three dimensions". Medical Engineering \& Physics, 2014.

[39] C. Milanese et al. "Anthropometry and body composition of female handball players according to competitive level or the playing position". Journal of Sport Sciencesi, vol.29, no.12, pp.301-1309, 2011.

[40] A.D. Stewart and L. Sutton, Body Composition in Sport, Exercise and Health. Abingdon, UK: Routledge, 2012.

[41] J. C. K. Wells et al. "BMI compared with 3-dimensional body shape: the UK National Sizing Survey". American Journal of Clinical Nutrition, vol.85, pp.419-425, 2007. 